\title{
Síndrome de Burnout e Rendimento Acadêmico em Estudantes da Primeira à Quarta Série de um Curso de Graduação em Medicina
}

\author{
Burnout Syndrome and Academic \\ Performance of First- and Second-Year \\ Medical Students
}

Mariana Ono Mori Tânia Cristina O. Valente ${ }^{I I}$ Luiz Fernando C. Nascimento ${ }^{I}$

\section{PALAVRAS-CHAVE \\ - Esgotamento Profissional. \\ - Estudantes de Medicina. \\ - Educação Médica.}

Recebido em: 15/08/2012

Aprovado em: 01/10/2012

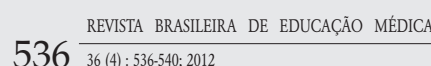

\footnotetext{
'Universidade de Taubaté, Taubaté, SP, Brasil.

"I Universidade Federal do Estado do Rio de Janeiro, Rio de Janeiro, RJ, Brasil.

The aim of this study was to to investigate the association between burnout syndrome and the academic performance of first and second year undergraduate medical students. The emotional commitment of the students varied according to which year or semester they were in, with significant differences for components of the syndrome among students with grades above and below the average, particularly for first year students. This indicates that in the course investigated the implementation of curricular reform has not added protection against burnout syndrome. An association was also found between the syndrome and the academic performance of first year students. Longitudinal studies into this subject are required in order to confirm the trends found and shed light on the interference of factors which could not be addressed in this preliminary study. 


\section{INTRODUÇÃO}

O ingresso no curso de Medicina requer a transformação precoce de adolescentes em profissionais, o que, muitas vezes, impõe adquirir maturidade para escolhas individuais no que diz respeito à atuação profissional e ao relacionamento com futuros colegas e pacientes ${ }^{1}$.

As repercussões desse processo de adaptação a novas situações foram descritas por Hans Selye ${ }^{2}$ como estresse, compreendendo três fases: de alerta, resistência e exaustão. Quando esgotado, este modelo trifásico de evolução (que Selye ${ }^{2}$ denomina "síndrome geral de adaptação") e sem o desenvolvimento de mecanismos de restauração, o organismo pode evoluir para o "distress" ou estresse destrutivo, com efeitos deletérios à saúde física e psicológica, que, no estágio crônico, podem evoluir para a Síndrome de Burnout.

Atualmente, a definição mais utilizada para burnout é a de Maslach e Jackson?: uma "síndrome multidimensional constituída por exaustão emocional, desumanização e reduzida realização pessoal no trabalho". A exaustão emocional se caracteriza pela sensação de esgotamento emocional e físico. A desumanização - despersonalização na versão de Maslach e Jackson $^{3}$, posteriormente denominada cinismo por Maslach ${ }^{4}$ - é marcada por uma atitude de distanciamento emocional com colegas e pacientes/clientes; os contatos se tornam impessoais, desprovidos de afetividade, desumanos; a reduzida realização pessoal diminui a satisfação, e a sensação de ineficiência no trabalho o torna um fardo.

A causa principal não é definida, existindo múltiplos fatores que facilitam o desencadeamento da Síndrome de Burnout. Em alunos do curso de graduação em Medicina, é grande a pressão para mostrar seu valor não apenas a si mesmos, mas também a terceiros. São estes — pacientes, colegas e a sociedade - que irão reconhecer e avaliar a atuação do profissional. E, em cada etapa a conquistar para se tornar um médico melhor, a tendência é o afunilamento do número de vagas disponíveis e o aumento da concorrência. Muitas vezes, nessa tentativa de querer sempre se destacar a fim de "queimar até a exaustão", estes estudantes podem ser grandes vítimas de burnout.

Em 2001, foram publicadas as Diretrizes Curriculares Nacionais para os Cursos de Graduação em Medicina ${ }^{5}$, que definiram princípios, fundamentos, condições e procedimentos necessários para que as escolas médicas, na sua condição formadora, se adequassem à realidade da saúde no Brasil. Estas diretrizes propõem a formação de um profissional com perfil atuante diante dos problemas de saúde da população, assim como diante dos problemas enfrentados pelo sistema de saúde, caracterizando a necessidade de um médico humanista e ético, com pensamento crítico e reflexivo, responsabilidade social e capacitado a atuar em todos os níveis de atenção.

Estas diretrizes exigiram mudanças curriculares e estruturais em todos os cursos de Medicina. Assim, em 2007, o Departamento de Medicina da Universidade de Taubaté (Unitau) implantou um novo modelo de currículo, que propôs integração e conectividade horizontal/vertical, eliminando conteúdos disciplinares isolados em sua quase totalidade, juntando-os em módulos. Criaram-se disciplinas - Bases Moleculares (composta por Bioquímica, Biofísica, Genética e Biologia Molecular), Bases Morfológicas (Anatomia Descritiva, Embriologia e Histologia), Bases da Relação Patógeno-Hospedeiro (Microbiologia, Imunologia, Moléstias Infecciosas e Parasitologia), Bases Fisiopatológicas Médicas (Fisiologia e Patologia) e Introdução à Prática Clínica (IPC I e II), estas últimas com o objetivo de que alunos da primeira e segunda série, respectivamente, vivenciassem mais precocemente a prática clínica no sistema de saúde ${ }^{6}$. O modelo que antecedia a reforma se apoiava numa visão centrada na racionalidade anatomoclínica, sendo os dois primeiros anos dedicados às disciplinas do ciclo básico, e os dois anos seguintes a disciplinas teóricas. Estas mudanças criaram uma situação de transição, com a coexistência de turmas no currículo novo (1 $1^{\mathrm{a}}, 2^{\mathrm{a}}$ e $3^{\mathrm{a}}$ séries) e no modelo antigo ( $4^{\underline{a}}$ série).

Tal circunstância motivou o estudo da prevalência de burnout nestes alunos, revelando a presença concomitante de médias acima dos valores das médias encontradas para as dimensões exaustão emocional (EE) e despersonalização (DP) e abaixo das médias para a sensação de competência como estudante $(\mathrm{RP})$ - definidora da Síndrome de Burnout - em 20\% dos alunos, sendo mais prevalente na quarta e na segunda série, relacionada a fatores como moradia, pensamentos sobre a possibilidade de desistência do curso e realização de atividade fí$\operatorname{sica}^{7}$. A pesquisa ainda mostrou que, embora a prevalência fosse maior na quarta série, inserida no currículo antigo, um número de fatores maior afetava os alunos da terceira série, a partir da qual foi implantado o novo modelo curricular. Dados da literatura ${ }^{8,9}$ sugerem que a organização do currículo, associada a fatores individuais, pode influenciar o nível de estresse entre estudantes de Medicina, podendo levar ao burnout.

O presente estudo foi realizado com o objetivo de analisar a associação entre a presença de burnout e o rendimento acadêmico dos estudantes de graduação em Medicina da Universidade de Taubaté.

\section{MÉTODO}

A pesquisa foi descritiva e retrospectiva, sendo a população constituída da totalidade de estudantes do primeiro ao quarto 
ano do curso de graduação em Medicina da Universidade de Taubaté (Unitau). Foi registrada e aprovada pelo Comitê de Ética da Pró-Reitoria de Pesquisa e Pós-Graduação da Unitau, sob o número 278/09.

Compreendeu duas etapas: na primeira, foram aplicados dois questionários autoinformados, um relacionado aos dados sociodemográficos, e o MBI-SS (Maslach Burnout Inventory Student Survey), com o objetivo de estudar a prevalência da Síndrome de Burnout entre estudantes da primeira à quarta série no curso de Medicina da Universidade de Taubaté (SP), realizada de agosto a dezembro de 2009 e cujos resultados são apresentados em outra publicação (Mori et al) ${ }^{7}$. Esta primeira pesquisa gerou como resultados as médias de cada aluno para cada uma das dimensões de burnout por série.

Na segunda etapa, destinada à avaliação do desempenho acadêmico, as notas de cada estudante da primeira à quarta série por disciplina e por série referentes ao ano letivo de 2009 foram obtidas na Secretaria do Departamento de Medicina, sendo analisadas de agosto a dezembro de 2010.

$\mathrm{O}$ rendimento acadêmico dos estudantes foi comparado mediante a divisão destes em dois grupos: o primeiro foi composto por alunos com presença concomitante de médias acima dos valores das médias encontradas para as dimensões exaustão emocional (EE) e despersonalização (DP) e abaixo das médias para a sensação de competência como estudante (RP) definida como burnout; o outro grupo incluiu os alunos sem burnout, ou seja, com médias abaixo dos valores das médias encontradas para as dimensões exaustão emocional (EE) e despersonalização (DP) e acima das médias para a sensação de competência como estudante (RP).

Em cada um desses dois grupos foram identificados os alunos cujas notas finais para cada uma das disciplinas analisadas estavam acima da média requerida pela universidade (média $=6,0$ ), assim como os estudantes cujas notas estavam abaixo da média $(5,99)$ em cada disciplina de cada uma das séries do curso, buscando-se identificar a associação entre a presença de burnout e o desempenho acadêmico dos estudantes.

As disciplinas em que havia apenas notas acima da média não foram analisadas, por impossibilidade comparativa. Para a análise dos dados foi utilizado o programa SPSS Statistics17.0, considerando-se significante um $\mathrm{p}<0,05$.

\section{RESULTADOS E DISCUSSÃO}

A Tabela 1 mostra os resultados referentes à primeira etapa do estudo com as médias para cada uma das dimensões da síndrome, assim como a prevalência de burnout por série.
TABELA 1

Prevalência de Síndrome de Burnout por série em alunos da primeira à quarta série de um curso de graduação em

Medicina, Taubaté (SP), 2009

\begin{tabular}{ccccccc}
\hline $\begin{array}{c}\text { Série do } \\
\text { curso de } \\
\text { Medicina }\end{array}$ & $\begin{array}{c}\text { Total de } \\
\text { alunos } \\
\text { partici- } \\
\text { pantes } \\
\text { por série }\end{array}$ & $\begin{array}{c}\text { Exaustão } \\
\text { emo- } \\
\text { cional }^{*}\end{array}$ & $\begin{array}{c}\text { Desperso- } \\
\text { nalização }\end{array}$ & $\begin{array}{c}\text { Reali- } \\
\text { zação } \\
\text { pessoal }\end{array}$ & $\begin{array}{c}\text { Número } \\
\text { de alunos } \\
\text { com } \\
\text { burnout }\end{array}$ & $\begin{array}{c}\text { Preva- } \\
\text { lência } \\
\text { (\%) }\end{array}$ \\
\hline 1 & 60 & 3,34 & 1,97 & 3,98 & 11 & 18,3 \\
2 & 61 & $3,14^{*}$ & 1,53 & 4,10 & 10 & 16,4 \\
3 & 62 & 3,52 & 1,84 & 3,79 & 14 & 22,5 \\
4 & 42 & $3,81^{*}$ & 1,64 & 4,21 & 11 & 26,1 \\
Total & 225 & $\mu=3,42$ & $\mu=1,75$ & $\mu=4,00$ & 46 & 20,4 \\
\hline
\end{tabular}

* $\mathrm{p}<0,05$ (obtido pela Anova)

A Tabela 2 mostra a comparação, por disciplina e por série, do número de alunos com notas acima e abaixo da média para a aprovação, no curso onde a pesquisa foi realizada (média igual ou superior a 6,0), assim como as médias das dimensões do burnout. Identificam-se, nas três primeiras séries do curso estudado, disciplinas onde há diferenças significativas entre os alunos com notas acima e abaixo da média, para as dimensões do burnout.

Na primeira série, na disciplina Bases Morfológicas, isto acontece para EE, RP, DP, enquanto na segunda série, para Fisiopatologia nas dimensões EE e RP. Na terceira série, quatro disciplinas (Clínica Médica, Farmacologia, Patologia e Semiologia) apresentam diferenças para RP, e na quarta série não há diferenças significativas.

A Tabela 3 mostra o estudo da associação entre a Síndrome de Burnout e as notas dos alunos para as disciplinas, por série, realizado por meio do Teste do X2. Para as disciplinas Bases Morfológicas, na primeira série, e Fisiopatologia, na segunda, constatou-se relação entre o rendimento acadêmico dos estudantes pesquisados e a presença da Síndrome de Burnout.

O interesse sobre a saúde psicológica do estudante de Medicina tem crescido a partir de meados da década de 1990. Entretanto, o único estudo publicado que abordou efeitos das mudanças curriculares nesta população é o de $\mathrm{Zuardi}^{10}{ }^{10}$ embora este autor tenha utilizado instrumento diverso e tenha focalizado a ansiedade e não a Síndrome de Burnout. Gonçalves ${ }^{8}$, ao discutir as propostas de mudança do ensino médico, propõe a inclusão de conteúdos relacionados às ciências sociais para a "humanização" dos teores essencialmente fundamentados no modelo biomédico no currículo das escolas. Entretanto, como ressalta Baldassin', o equilíbrio psicoemocional dos estudantes está além da substituição de modelos e atitudes adquiridos antes da entrada na universidade ou observados, sofridos, reprimidos e estimulados durante o curso. 
TABELA 2

Médias das dimensões do burnout por disciplina e por série em alunos da primeira à quarta série de um curso de graduação em Medicina, Taubaté (SP), 2009

\begin{tabular}{|c|c|c|c|c|c|c|c|c|c|c|c|}
\hline & \multirow{2}{*}{ Dimensões } & \multicolumn{4}{|c|}{ Exaustão emocional } & \multicolumn{3}{|c|}{ Descrença } & \multicolumn{3}{|c|}{ Competência como estudante } \\
\hline & & $\mathbf{N}$ & m & $\mathrm{dp}$ & $\mathbf{P}$ & $\mathrm{m}$ & $\mathrm{dp}$ & $\mathrm{p}$ & m & $\mathrm{dp}$ & $\mathrm{p}$ \\
\hline \multirow{6}{*}{ 1a série } & \multirow{2}{*}{ Bases moleculares } & abaixo 20 & 3,62 & 0,88 & 0,135 & 2,52 & 1,76 & $0,004^{*}$ & 3,59 & 1,33 & $0,036^{*}$ \\
\hline & & acima 18 & 3,10 & 1,20 & 0,142 & 1,04 & 1,04 & $0,003^{*}$ & 4,42 & 0,95 & $0,034^{*}$ \\
\hline & \multirow{2}{*}{ Bases morfológicas } & abaixo 15 & 3,88 & 0,78 & $0,016^{*}$ & 2,90 & 1,82 & $0,000^{*}$ & 3,41 & 1,35 & $0,016^{*}$ \\
\hline & & acima 23 & 3,04 & 1,11 & $0,010^{*}$ & 1,12 & 1,02 & $0,003^{*}$ & 4,37 & 0,99 & $0,027^{*}$ \\
\hline & \multirow{2}{*}{ IPC-I } & abaixo 1 & 5,20 & & 0,087 & 5,50 & & $0,011^{*}$ & 0,00 & & $0,000^{*}$ \\
\hline & & acima 36 & 3,35 & 1,04 & & 1,62 & 1,43 & & 4,16 & 0,99 & \\
\hline \multirow{6}{*}{$2^{a}$ série } & \multirow{2}{*}{$\mathrm{RPH}$} & abaixo 4 & 3,40 & 1,39 & 0,704 & 2,88 & 1,16 & $0,048^{*}$ & 3,37 & 0,81 & 0,165 \\
\hline & & acima 53 & 3,16 & 1,19 & 0,760 & 1,48 & 1,34 & 0,090 & 4,15 & 1,07 & 0,150 \\
\hline & \multirow{2}{*}{ Fisiopatologia } & abaixo12 & 3,91 & 1,08 & $0,014^{*}$ & 2,08 & 1,24 & 0,149 & 3,65 & 1,17 & 0,109 \\
\hline & & acima 45 & 2,98 & 1,15 & $0,017^{*}$ & 1,44 & 1,38 & 0,136 & 4,21 & 1,02 & 0,152 \\
\hline & \multirow{2}{*}{ IPC-II } & abaixo 3 & 3,67 & 0,70 & 0,471 & 2,42 & 2,02 & 0,277 & 3,66 & 2,02 & 0,482 \\
\hline & & acima 54 & 3,15 & 1,21 & 0,332 & 1,53 & 1,33 & 0,527 & 4,12 & 1,02 & 0,737 \\
\hline \multirow{14}{*}{ 3a série } & \multirow{2}{*}{ Anatomia Topográfica } & abaixo 11 & 3,34 & 1,78 & 0,581 & 1,88 & 1,31 & 0,867 & 3,70 & 1,11 & 0,573 \\
\hline & & acima 46 & 3,56 & 0,96 & 0,705 & 1,81 & 1,37 & 0,865 & 3,90 & 1,04 & 0,594 \\
\hline & \multirow{2}{*}{ Clínica Médica } & abaixo 17 & 3,54 & 1,13 & 0,926 & 2,15 & 1,41 & 0,243 & 3,09 & 0,84 & $0,000^{*}$ \\
\hline & & acima 40 & 3,51 & 1,17 & 0,926 & 1,69 & 1,31 & 0,261 & 4,19 & 0,97 & $0,000^{*}$ \\
\hline & \multirow{2}{*}{ Farmacologia } & abaixo 17 & 3,22 & 0,89 & 0,238 & 1,85 & 1,12 & 0,885 & 3,38 & 1,13 & $0,018^{*}$ \\
\hline & & acima 39 & 3,62 & 1,23 & 0,184 & 1,79 & 1,46 & 0,872 & 4,10 & 0,95 & $0,030^{*}$ \\
\hline & \multirow{2}{*}{ Obstetrícia } & abaixo 2 & 3,70 & 1,27 & 0,823 & 1,75 & 1,06 & 0,937 & 3,08 & 0,82 & 0,293 \\
\hline & & acima 55 & 3,51 & 1,15 & 0,870 & 1,83 & 1,36 & 0,935 & 3,89 & 1,05 & 0,390 \\
\hline & \multirow{2}{*}{ Patologia } & abaixo 9 & 3,58 & 1,54 & 0,870 & 2,36 & 1,70 & 0,196 & 2,91 & 0,99 & $0,002^{*}$ \\
\hline & & acima 48 & 3,51 & 1,08 & 0,900 & 1,72 & 1,23 & 0,310 & 4,04 & 0,97 & $0,009^{*}$ \\
\hline & \multirow{2}{*}{ Puericultura e Pediatria } & abaixo 1 & 3,80 & & 0,808 & 1,75 & & 0,956 & 4,83 & . & 0,356 \\
\hline & & acima 56 & 3,51 & 1,16 & & 1,83 & 1,36 & . & 3,84 & 1,05 & \\
\hline & \multirow{2}{*}{ Semiologia } & abaixo 10 & 3,42 & 1,28 & 0,766 & 2,27 & 1,35 & 0,248 & 3,25 & 0,69 & $0,043^{*}$ \\
\hline & & acima 47 & 3,54 & 1,13 & 0,788 & 1,73 & 1,34 & 0,266 & 3,99 & 1,07 & $0,013^{*}$ \\
\hline \multirow{8}{*}{$4^{\mathrm{a}}$ série } & \multirow{2}{*}{ Clínica Cirúrgica II } & abaixo 6 & 3,87 & 1,44 & 0,933 & 2,04 & 1,54 & 0,359 & 3,83 & 0,69 & 0,292 \\
\hline & & acima 30 & 3,91 & 1,18 & 0,943 & 1,56 & 1,08 & 0,492 & 4,33 & 1,09 & 0,176 \\
\hline & \multirow{2}{*}{ Pediatria e Adolescência } & abaixo 2 & 3,40 & 1,41 & 0,550 & 2,62 & 1,24 & 0,221 & 3,58 & 0,35 & 0,361 \\
\hline & & acima 34 & 3,93 & 1,21 & 0,687 & 1,58 & 1,15 & 0,437 & 4,29 & 1,06 & 0,132 \\
\hline & \multirow{2}{*}{ Urologia } & abaixo 2 & 4,10 & 0,42 & 0,819 & 2,25 & 1,77 & 0,452 & 4,42 & 0,82 & 0,820 \\
\hline & & acima 34 & 3,89 & 1,24 & 0,627 & 1,60 & 1,14 & 0,696 & 4,24 & 1,07 & 0,815 \\
\hline & \multirow{2}{*}{ Anestesiologia e Terapia da Dor } & abaixo 25 & 3,91 & 1,22 & 0,962 & 1,58 & 1,12 & 0,653 & 4,08 & 1,07 & 0,144 \\
\hline & & acima 11 & 3,89 & 1,23 & 0,963 & 1,77 & 1,29 & 0,674 & 4,63 & 0,91 & 0,125 \\
\hline
\end{tabular}

${ }^{*} \mathrm{P}<0,05$

TABela 3

Comparação de presença de Síndrome de Burnout entre alunos da primeira à quarta série de um curso de graduação em Medicina, por disciplina e por série, Taubaté (SP), 2009

\begin{tabular}{|c|c|c|c|c|}
\hline & \multirow{2}{*}{ Disciplinas } & \multicolumn{2}{|c|}{ Síndrome de Burnout } & \multirow{2}{*}{$\mathrm{p}$} \\
\hline & & Presente & Ausente & \\
\hline \multirow[t]{3}{*}{ 1'a série } & Bases moleculares & 8 & 30 & 0,15 \\
\hline & Bases morfológicas & 8 & 30 & $0,02^{*}$ \\
\hline & Introd. Prática Clínica I & 8 & 29 & 0,48 \\
\hline \multirow[t]{3}{*}{ 2a série } & Bases da Relação Patógeno-Hospedeiro & 13 & 44 & 1,00 \\
\hline & Fisiopatologia & 13 & 44 & $0,03^{*}$ \\
\hline & Introd. Prática Clínica II & 13 & 44 & 0,24 \\
\hline \multirow[t]{7}{*}{ 3a série } & Anatomia Topográfica & 13 & 44 & 0,99 \\
\hline & Clínica Médica & 13 & 44 & 0,66 \\
\hline & Farmacologia & 13 & 44 & 0,34 \\
\hline & Obstetrícia & 13 & 44 & 1,00 \\
\hline & Patologia & 13 & 44 & 1,00 \\
\hline & Puericultura e Pediatria & 13 & 44 & 1,00 \\
\hline & Semiologia & 13 & 44 & 0,85 \\
\hline \multirow[t]{4}{*}{$4^{a}$ série } & Clínica Cirúrgica II & 10 & 26 & 0,40 \\
\hline & Pediatria e Adolescência & 10 & 26 & 1,00 \\
\hline & Urologia & 10 & 26 & 1,00 \\
\hline & Anestesiologia e Terapia da Dor & 10 & 26 & 1,00 \\
\hline
\end{tabular}

$\mathrm{P}<0,05\left(\mathrm{X}^{2}\right)$ 
Outro fato a enfatizar nos resultados deste estudo é que uma das disciplinas onde houve associação entre Síndrome de Burnout e rendimento acadêmico dos estudantes foi criada com o novo currículo. Um das explicações para isto é a possibilidade de que as propostas de mudança curricular não tenham sido acompanhadas de uma satisfatória capacitação de docentes. Como citado por Almeida ${ }^{11}$, no processo de reforma curricular, é exigido do docente um papel mais cooperativo, em busca de uma facilitação do processo ensino-aprendizagem e de maior integração com outras disciplinas da área da saúde, seja pelo planejamento, seja pela execução das tarefas. Tais exigências geralmente provocam sensações de insegurança e perda de poder no médico docente, acarretando uma resistência a este processo de mudança. Costa et al. ${ }^{12}$ encontraram associação potencial entre a Síndrome de Burnout e três variáveis referentes ao processo educacional: falta de confiança na aquisição de habilidades, sensação de desconforto em atividades acadêmicas e falta de prazer nas atividades relacionadas ao curso. Tais achados reforçam a responsabilidade dos docentes e da coordenação dos cursos de graduação em Medicina no que diz respeito à saúde emocional dos estudantes.

\section{CONCLUSÃO}

Os resultados apresentados mostram que, no curso estudado, o comprometimento emocional dos alunos variou conforme a série, com diferenças significativas para os componentes da síndrome entre alunos com notas acima e abaixo da média, sendo o primeiro ano o mais afetado. Isto indica que, neste curso, a implantação da reforma curricular não foi um fator de proteção para a Síndrome de Burnout. Houve também associação entre a síndrome e o rendimento acadêmico dos estudantes em disciplinas da primeira e da segunda série.

Estudos longitudinais são imprescindíveis para confirmar as tendências encontradas e esclarecer a interferência de fatores que não puderam ser abordados neste estudo, considerado preliminar.

\section{REFERÊNCIAS}

1. Shaw D, Wedding D, Zeldow P, Diehl N. Special problems of medical students. Part 1: The Physician and Society [S.1.:s.n.]:67-84. 2002.

Selye H. The stress of life. New York: McGraw Hill; 1984.

2. Maslach C, Jackson S. Maslach Burnout Inventory, Manual. Consulting Psychologists. Palo Alto: University of California; 1986.

3. Maslach C, Jackson SE, Leiter MP. The Maslach Burnout Inventory, Manual. Consulting Psycologists. Palo Alto: University of California; 1996.
4. Brasil. Ministério da Educação. Conselho Nacional de Educação. Câmara de Educação Superior. Resolução CNE/CES nº 4 de 7 de novembro de 2001. Institui diretrizes curriculares nacionais do curso de graduação em Medicina. Diário Oficial da União. Brasília, 9 nov. 2001; Seção 1, p.38.

5. Universidade de Taubaté. Departamento de Medicina. Comissão Permanente de Estudo Curricular. Proposta de Mudança Curricular do Depto. de Medicina da Universidade de Taubaté. Taubaté, s.d.

6. Mori MO, Valente TCO, Nascimento LFC. Burnout Syndrom and Associated Factors Among Medical Students. Rev Bras Psiquiatr. 2012. In press.

7. Gonçalves MB, Benevides-Pereira AMT. Considerações sobre o ensino médico no Brasil: conseqüências afetivo emocionais nos estudantes. Rev Bras.Educ Med. 2009; 33(3):493-504

8. Baldassin S. Ansiedade e Depressão no Estudante de Medicina: Revisão de Estudos Brasileiros. Cadernos ABEM. 2010; 6:19-26.

9. Zuardi AW, Prota FDG, Del-Bem CM. Reduction of the anxiety of medical students after currcular reform. Rev Bras Psiquiatr. 2008; 30(2):136-8.

10. Almeida HGG, Ferreira Filho OF. Educação permanente de docentes: análise crítica de experiências não sistematizadas. Rev Bras Educ Méd. 2008; 32 (2): 240-247.

11. Costa EFO, Santos AS, Santos ATRA, Melo EV, Andrade TM. Burnout Syndrome and associated factors among medical students: a cross sectional study. Clinics. 2012; 67(6):573-579

\section{CONTRIBUIÇÃO DOS AUTORES}

Tania C. O. Valente e Mariana Ono Mori participaram da concepção do estudo, coleta, análise de dados e elaboração do artigo. Luiz Fernando C. Nascimento participou da análise de dados e elboração do artigo.

\section{CONFLITO DE INTERESSES}

Declarou não haver.

\section{ENDEREÇO PARA CORRESPONDÊNCIA}

Tânia C. Oliveira Valente

Rua Alexandre Fleming, 160

Jardim Maria Augusta

CEP: 12070002 — Taubaté, SP.

E-mail: taniaunitau@gmail.com 